\title{
Impact of Body Mass Index on Short-Term Outcomes in Patients Undergoing Percutaneous Coronary Intervention in Newfoundland and Labrador, Canada
}

\author{
Anne B. Gregory, ${ }^{1,2}$ Kendra K. Lester, ${ }^{2}$ Deborah M. Gregory, ${ }^{2,3}$ Laurie K. Twells, ${ }^{2,4}$ \\ William K. Midodzi, ${ }^{2}$ and Neil J. Pearce ${ }^{1,3}$ \\ ${ }^{1}$ Eastern Health, St. John's, NL, Canada A1B 3V6 \\ ${ }^{2}$ Department of Clinical Epidemiology, Faculty of Medicine, Memorial University of Newfoundland, St. John's, NL, Canada A1B 3 V6 \\ ${ }^{3}$ Department of Medicine, Faculty of Medicine, Memorial University of Newfoundland, St. John's, NL, Canada A1B 3V6 \\ ${ }^{4}$ School of Pharmacy, Memorial University of Newfoundland, St. John's, NL, Canada A1B 3V6
}

Correspondence should be addressed to Deborah M. Gregory; dgregory@mun.ca

Received 27 May 2016; Revised 21 July 2016; Accepted 25 July 2016

Academic Editor: Mariantonietta Cicoira

Copyright (C) 2016 Anne B. Gregory et al. This is an open access article distributed under the Creative Commons Attribution License, which permits unrestricted use, distribution, and reproduction in any medium, provided the original work is properly cited.

\begin{abstract}
Background and Aim. Obesity (BMI $\left.\geq 30 \mathrm{~kg} / \mathrm{m}^{2}\right)$ is associated with advanced cardiovascular disease requiring procedures such as percutaneous coronary intervention (PCI). Studies report better outcomes in obese patients having these procedures but results are conflicting or inconsistent. Newfoundland and Labrador (NL) has the highest rate of obesity in Canada. The aim of the study was to examine the relationship between BMI and vascular and nonvascular complications in patients undergoing PCI in NL. Methods. We studied 6473 patients identified in the APPROACH-NL database who underwent PCI from May 2006 to December 2013. BMI categories included normal, $18.5 \leq \mathrm{BMI}<25.0(n=1073)$; overweight, $25.0 \leq \mathrm{BMI}<30(n=2608)$; and obese, BMI $\geq 30.0$ $(n=2792)$. Results. Patients with obesity were younger and had a higher incidence of diabetes, hypertension, and family history of cardiac disease. Obese patients experienced less vascular complications (normal, overweight, and obese: 8.2\%, 7.2\%, and 5.3\%, $p=0.001)$. No significant differences were observed for in-lab $(4.0 \%, 3.3 \%$, and $3.1 \%, p=0.386)$ or postprocedural $(1.0 \%, 0.8 \%$, and $0.9 \%, p=0.725)$ nonvascular complications. After adjusting for covariates, BMI was not a significant factor associated with adverse outcomes. Conclusion. Overweight and obesity were not independent correlates of short-term vascular and nonvascular complications among patients undergoing PCI.
\end{abstract}

\section{Introduction}

Obesity is an independent risk factor for cardiovascular disease $[1-5]$ and is associated with advanced cardiovascular disease requiring procedures such as percutaneous coronary intervention (PCI) and coronary artery bypass grafting (CABG), reduction in life expectancy [6], and a higher mortality rate $[3,7,8]$. A number of observational studies have reported improved clinical outcomes (i.e., increased survival benefit) in overweight and obese patients treated for cardiovascular diseases compared to normal weight patients, a phenomenon commonly referred to as "obesity paradox"
[9-14]. This phenomenon is considered to be counterintuitive, referred to as "reverse epidemiology," and reported in patients with hypertension [15], heart failure [16], coronary artery disease (CAD) [15, 17-19], CABG [17-20], and PCI [17-19]. The term "reverse epidemiology" was first used in a 2003 study by Kalantar-Zadeh et al. [21] which focused on cardiovascular risk factors including body mass index (BMI), serum cholesterol, and blood pressure in maintenance hemodialysis patients. Obesity, hypercholesterolemia, and hypertension were paradoxically associated with survival, the opposite of that observed in the general population. In 2004, Kalantar-Zadeh et al. also reported a protective role 
of conventional cardiovascular risk factors in chronic heart failure [22]. Use of the term "reverse epidemiology" and alternative terms including "risk factor reversal" and "altered risk factor patterns" [23] has continued in the research literature; however, some refer to the term as confusing, confounding, and inaccurate [24] and suggest it may be a "questionable concept." Inconsistent results have been reported regarding the association between BMI and short-term clinical outcomes (i.e., vascular complication, nonvascular in-lab and postprocedural complications) and/or mortality in patients undergoing PCI $[9,10,13,25-31]$; therefore, it is not entirely clear whether an obesity paradox exists.

Obesity is a common and rapidly growing public health concern. Between 1985 and 2011, the prevalence of this disease in Canada increased by $200 \%$ from $6.1 \%$ to $18.3 \%$ equating to more than 4.8 million adults, with continued increases projected [32]. Newfoundland and Labrador (NL) has the highest rate of obesity in Canada. It is estimated that $71 \%$ of the province's population will be either overweight or obese by 2019 [32]. There is a paucity of data on the prevalence of obesity in patients undergoing PCI in the province. Furthermore, the relationship between short-term clinical outcomes and BMI has not been examined in patients undergoing PCI in NL. In the present study, we (1) examine the prevalence of obesity among patients undergoing PCI and the differences among BMI groups on demographic, clinical, and procedural findings and (2) examine the association between the most commonly used anthropometric parameter to assess adiposity (i.e., BMI) and short-term outcomes (vascular complication, nonvascular in-lab and postprocedural complications occurring within 48 hours).

\section{Methods}

2.1. Study Design. We performed a retrospective analysis of prospectively collected deidentified data for all patients 18 years of age and older who had a PCI between May 1, 2006, and December 31, 2013, in the province of NL, Canada, using a well-established clinical database (i.e., Alberta Provincial Project for Outcome Assessment in Coronary Heart DiseaseNewfoundland and Labrador (APPROACH-NL)). Detailed prospective demographic, clinical, and procedural data on all patients undergoing diagnostic cardiac catheterization and/or percutaneous coronary intervention (PCI) and cardiac surgery since 2006 is collected by specifically trained clinical cardiac catheterization database nurses. Nurses collect and record, on an abstraction sheet, patient data provided by nurses responsible for the care of the patient which includes examination and assessment of the access site for potential vascular complications. The attending physician also examined the vascular access site. All data are verified by chart review until hospital discharge by these nurses. Prospectively collected data on each consecutive patient is entered into the APPROACH-NL clinical database. A research nurse is responsible for the management of the database including completeness of data entry and quality assurance activities. Details of the database and methods of collection have been previously described [33]. If patients are not hospitalized, they remain in the local area for 24 hours and are advised to return to the emergency department (ER) if they encounter any problems. ER admissions are audited by a clerk in the cardiac catheterization laboratory in the event that a patient returns to the ER.

2.2. Study Population. For the current study, all consecutive PCIs $(N=6633)$ performed on patients 18 years of age and older between May 1, 2006, and December 31, 2013, at the Health Science Centre, Eastern Health, NL, were enrolled. PCI procedures performed on underweight (BMI $\left.<18.5 \mathrm{~kg} / \mathrm{m}^{2}\right)$ individuals $(n=47)$ or those with missing BMI data or unlikely valid BMI levels of $>70$ or $<11 \mathrm{~kg} / \mathrm{m}^{2}(n=$ 113) were excluded. The remaining patients comprised the study cohort. Based on these selection criteria, 6,473 patients were included in the final analysis.

Weight and height were measured and documented by a nurse at the time of PCI. If patients were unstable, selfreported weight and height were collected and BMI was calculated. Patients were grouped according to three BMI categories using the World Health Organization classification system: normal $\left(18.5-24.9 \mathrm{~kg} / \mathrm{m}^{2}\right)$, overweight $(25.0-$ $\left.29.9 \mathrm{~kg} / \mathrm{m}^{2}\right)$, and obese $\left(\geq 30 \mathrm{~kg} / \mathrm{m}^{2}\right)$ [34]. These categorizations reflect relative increasing levels of risk to health [35].

2.3. Clinical Outcomes and Definitions. The primary outcome was short-term complications occurring within 48 hours after the intervention. The clinical definitions for complications were as follows. Vascular access complications were defined as hematoma $(>5 \mathrm{~cm})$, pseudoaneurysm, arteriovenous fistula, vascular occlusion, access site bleeding, retroperitoneal bleed, loss of distal pulse, or occlusion. Nonvascular complications included in-lab events (abrupt coronary closure, emergency CABG, access site complications, death, ventricular tachycardia/ventricular fibrillation, pulmonary edema, shock, and dissection) and postprocedural complications included death, myocardial infarction, emergency CABG, abrupt coronary closure, hemorrhagic or ischemic CVA, and GI bleed. Each of the outcomes was a composite of the individual outcomes defined in each category.

2.4. Ethical Considerations. All patients who had a PCI during the time period under examination gave written informed consent to the cardiac care program for data collection and follow-up observation after PCI. The study protocol was approved by the Health Research Ethics Authority of Memorial University and Eastern Health.

2.5. Data Analysis. Demographic characteristics and clinical and procedural related variables were summarized. Continuous variables were expressed as mean \pm standard deviation (SD). Categorical variables were expressed as frequencies and percentages. Continuous variables were compared using ANOVA, and the differences between categorical variables were examined using the $\chi^{2}$ test and, where appropriate, 
TABLE 1: Baseline characteristics of patients according to categories of BMI.

\begin{tabular}{|c|c|c|c|c|c|}
\hline Variable & Total $N$ & NW & OW & $\mathrm{OB}$ & $p$ value $^{*}$ \\
\hline Number of patients & 6473 & 1073 & 2608 & 2792 & \\
\hline Age, years & 6473 & $65.1 \pm 11.1$ & $63.1 \pm 10.5$ & $60.7 \pm 10.1$ & $p<0.001$ \\
\hline Male sex & 6473 & $695(64.8)$ & $1975(75.7)$ & $1945(69.7)$ & $p<0.001$ \\
\hline \multicolumn{6}{|c|}{ Cardiovascular risk factors } \\
\hline HTN & 6462 & $658(61.4)$ & $1661(63.8)$ & $2066(74.1)$ & $p<0.001$ \\
\hline Hyperlipidemia & 6462 & $905(84.4)$ & $2241(86.1)$ & $2434(87.4)$ & $p=0.050$ \\
\hline Diabetes & 6464 & $226(21.1)$ & $637(24.5)$ & $1040(37.3)$ & $p<0.001$ \\
\hline Family history & 6440 & $622(58.3)$ & $1627(62.7)$ & $1822(65.5)$ & $p<0.001$ \\
\hline Smoking status & 6421 & & & & \multirow{3}{*}{$p=0.441$} \\
\hline Never & 1719 & $298(28.1)$ & $698(27.0)$ & $723(26.1)$ & \\
\hline Smoking history & 4702 & $763(71.9)$ & $1891(73.0)$ & $2048(73.9)$ & \\
\hline PVD & 6460 & $91(8.5)$ & $172(6.6)$ & $156(5.6)$ & $p=0.005$ \\
\hline COPD & 6459 & $156(14.6)$ & $335(12.9)$ & 479 (17.2) & $p<0.001$ \\
\hline
\end{tabular}

Values are presented as $n(\%)$ or mean $\pm \mathrm{SD}$, as indicated.

* $p$ values for chi-squared or ANOVA tests.

BMI: body mass index; COPD: chronic obstructive pulmonary disease; NW: normal weight; OB: obese; OW: overweight; HTN: hypertension; PVD: peripheral vascular disease.

TABLE 2: Medications at time of referral for PCI by BMI category.

\begin{tabular}{|c|c|c|c|c|c|}
\hline & Total $N$ & NW & OW & $\mathrm{OB}$ & $p$ value $^{*}$ \\
\hline Number of patients & 6473 & 1073 & 2608 & 2792 & \\
\hline Beta blockers & 6431 & $863(81.2)$ & $2154(83.1)$ & $2318(83.5)$ & $p=0.220$ \\
\hline ACE inhibitors & 6429 & $510(48.0)$ & $1296(50.0)$ & $1476(53.2)$ & $p=0.006$ \\
\hline ARB antagonist & 6428 & $104(9.8)$ & $305(11.8)$ & $431(15.5)$ & $p<0.001$ \\
\hline $\mathrm{CCB}$ & 6430 & $173(16.3)$ & $433(16.7)$ & $596(21.5)$ & $p<0.001$ \\
\hline LA nitrates & 6430 & 307 (28.9) & $729(28.1)$ & $869(31.3)$ & $p=0.032$ \\
\hline Statin therapy & 6427 & $874(82.3)$ & $2199(84.9)$ & $2345(84.5)$ & $p=0.134$ \\
\hline Aspirin & 6432 & $983(92.5)$ & $2402(92.6)$ & $2612(94.1)$ & $p=0.058$ \\
\hline Ticlopidine/clopidogrel & 6432 & $806(75.8)$ & $1846(71.2)$ & $1925(69.3)$ & $p<0.001$ \\
\hline Coumadin & 6429 & $15(1.4)$ & $47(1.8)$ & $59(2.1)$ & $p=0.329$ \\
\hline GP IIb/IIIa inhibitors & 6437 & $5(0.5)$ & $14(0.5)$ & $13(0.5)$ & $p=0.924$ \\
\hline LMWH & 6439 & $428(40.2)$ & $971(37.4)$ & $1003(36.1)$ & $p=0.068$ \\
\hline IV heparin & 6439 & $220(20.6)$ & $497(19.1)$ & $579(20.8)$ & $p=0.268$ \\
\hline IV nitrates & 6430 & $141(13.3)$ & $313(12.1)$ & 307 (11.1) & $p=0.149$ \\
\hline
\end{tabular}

Values are presented as $n(\%)$.

* $p$ values for chi-squared tests.

ACE: angiotensin converting enzyme; ARB: angiotensin receptor blocker; BMI: body mass index; CCB: calcium channel blockers; LA nitrates: long-acting nitrates; LMWH: low molecular weight heparin; NW: normal weight; OB: obese; OW: overweight; PCI: percutaneous coronary intervention.

the Fisher exact test is reported. All $p$ values were twotailed, with statistical significance defined by a $p$ value $<$ 0.05. Comparisons were performed for a trend in increasing BMI categories using $\chi^{2}$ test for trends. Univariate logistic regression analysis was performed to determine the odds ratio for vascular complications and nonvascular complications occurring in the cardiac care laboratory identified within 24-48 hours after PCI. Multivariate logistic regression analysis was used to examine independent predictors for each of the patient outcomes. Due to the low nonvascular postprocedural complication event rate, regression analyses were not performed. Variables identified in Tables 1-3 were selected for these models based on univariate $p$ values $<0.20$ and overall clinical significance. All statistical analyses were performed using IBM SPSS Statistics for Windows, Version 22.0 (IBM Corp., Armonk, NY) [36].

\section{Results}

A cohort of 6,473 patients was identified from the population of patients who had a PCI during the time period under examination. BMI values for normal weight, overweight, and obese patients from 2006 to 2013 are presented in Figure 1. Tables 1-3 show the baseline characteristics of patients according to categories of BMI, medications at 
TABLE 3: Admitting clinical, angiographic, and procedural data for patients undergoing PCI according to BMI category.

\begin{tabular}{|c|c|c|c|c|c|}
\hline & Total $N$ & NW & OW & $\mathrm{OB}$ & $p$ value $^{*}$ \\
\hline Number of patients & 6473 & $n=1073$ & $n=2608$ & $n=2792$ & \\
\hline \multicolumn{6}{|l|}{ Cardiovascular history } \\
\hline Prior PCI & 6462 & $226(21.1)$ & $543(20.9)$ & $627(22.5)$ & $p=0.318$ \\
\hline Prior CABG & 6462 & $129(12.0)$ & $298(11.5)$ & $289(10.4)$ & $p=0.247$ \\
\hline Prior HF & 6462 & $52(4.9)$ & $83(3.2)$ & $106(3.8)$ & $p=0.052$ \\
\hline Prior MI & 6462 & $212(19.8)$ & $527(20.3)$ & $581(20.8)$ & $p=0.734$ \\
\hline CVD & 6450 & $89(8.3)$ & $160(6.2)$ & $174(6.3)$ & $p=0.041$ \\
\hline Same sitting angioplasty & 6473 & $864(80.5)$ & $2138(82.0)$ & $2321(83.1)$ & $p=0.149$ \\
\hline IABP/cardiogenic shock & 6461 & $7(0.7)$ & $16(0.6)$ & $31(1.1)$ & $p=0.104$ \\
\hline Priority & 6466 & & & & \multirow{4}{*}{$p<0.001$} \\
\hline Low risk & 1861 & $220(20.5)$ & $783(30.0)$ & $858(30.7)$ & \\
\hline Emergency & 397 & $72(6.7)$ & $170(6.5)$ & $155(5.6)$ & \\
\hline Urgent & 4208 & $780(72.7)$ & $1654(63.4)$ & $1774(63.5)$ & \\
\hline $\mathrm{PE}$ & 6216 & $8(0.8)$ & $13(0.5)$ & $15(0.6)$ & $p=0.641$ \\
\hline Thromboembolic history & 6218 & $5(0.5)$ & $12(0.5)$ & $10(0.4)$ & $p=0.797$ \\
\hline DVT & 6219 & $16(1.6)$ & $32(1.3)$ & $32(1.2)$ & $p=0.663$ \\
\hline Stable angina & 1748 & $202(18.9)$ & $731(28.1)$ & $815(29.3)$ & $p<0.001$ \\
\hline ACS & 4341 & $n=791$ & $n=1724$ & $n=1825$ & \\
\hline STEMI & 1227 & $251(31.7)$ & $501(29.1)$ & $475(26.0)$ & \\
\hline Non-STEMI & 1939 & $349(44.1)$ & $787(45.6)$ & $803(44.0)$ & $p=0.001$ \\
\hline Unstable angina & 1174 & $191(24.1)$ & $436(25.3)$ & $547(30.0)$ & \\
\hline Thrombolytics contraindicated & 4143 & $11(1.5)$ & $27(1.6)$ & $24(1.4)$ & $p=0.831$ \\
\hline Failed thrombolysis & 4274 & $26(3.4)$ & $77(4.5)$ & $59(3.3)$ & $p=0.122$ \\
\hline \multicolumn{6}{|l|}{ Access site } \\
\hline Radial/brachial & 1172 & $193(18.0)$ & $468(17.9)$ & $511(18.3)$ & \multirow{2}{*}{$p=0.938$} \\
\hline Femoral & 5301 & $880(82.0)$ & $2140(82.1)$ & $2282(81.7)$ & \\
\hline \multicolumn{6}{|l|}{ Sheath size } \\
\hline Sheath size $5 \mathrm{Fr}$ & 924 & $160(14.9)$ & $393(15.1)$ & $371(13.3)$ & \multirow{3}{*}{$p=0.386$} \\
\hline Sheath size $6 \mathrm{Fr}$ & 5432 & $893(83.2)$ & $2171(83.3)$ & $2368(84.9)$ & \\
\hline Sheath size $7 / 8 \mathrm{Fr}$ & 114 & $20(1.9)$ & $43(1.6)$ & $51(1.8)$ & \\
\hline Closure device & 6470 & $484(45.1)$ & $1359(52.1)$ & $1506(54.0)$ & $p<0.001$ \\
\hline GP IIb/IIIa inhibitors & 6471 & $134(12.5)$ & $338(13.0)$ & $351(12.6)$ & $p=0.889$ \\
\hline
\end{tabular}

Values are presented as $n(\%)$.

${ }^{*} p$ values for chi-squared tests.

ACS = acute coronary syndrome; BMI: body mass index; CABG: coronary artery bypass grafting; CVD: cerebrovascular disease; DVT= deep vein thrombosis; HF: heart failure; IABP: intra-aortic balloon pump; MI: myocardial infarction; NW: normal weight; OB: obese; OW: overweight; PCI: percutaneous coronary intervention; PE: pulmonary embolism; PVD: peripheral vascular disease; STEMI: ST elevation myocardial infarction.

time of referral, and admitting clinical angiographic and procedural data.

3.1. Baseline Characteristics. Of the 6,473 patients, $16.6 \%$ were of normal weight $(n=1073), 40.3 \%$ were overweight $(n=2608)$, and $43.1 \%$ were obese $(n=2792)$. In each of the years examined, less than $19 \%$ of patients who had a PCI were of normal weight (Table 1 and Figure 1). The baseline characteristics of the study patients according to the three BMI categories are presented in Table 1. There were statistically significant differences between the groups on a number of characteristics. A higher proportion of overweight patients were male. Patients with obesity were younger, had a higher incidence of coronary risk factors such as diabetes mellitus and hypertension, and had a family history of coronary artery disease. Patients with a higher BMI were also more likely to have COPD, whereas normal weight patients were more likely to have PVD. No significant differences were observed in smoking status.

Medications at the time of referral for PCI were examined. The details regarding the use of medications prior to PCI are presented in Table 2. No significant differences were found in the use of acetylsalicylic acid, coumadin, preprocedural GP IIb/IIIa inhibitors, beta blockers, LMWH, IV heparin, IV nitrates, or statin therapy between the groups. Patients with obesity were less likely to receive the antiplatelet medication ticlopidine/clopidogrel but were more likely to receive an 
TABLE 4: Vascular and nonvascular complications occurring within 24 to 48 hours in patients undergoing PCI according to BMI category.

\begin{tabular}{|c|c|c|c|c|}
\hline & $\begin{array}{c}\text { NW } \\
(n=1073)\end{array}$ & $\begin{array}{c}\text { OW } \\
(n=2608) \\
\end{array}$ & $\begin{array}{c}\text { OB } \\
(n=2792) \\
\end{array}$ & $p$ value ${ }^{*}$ \\
\hline Vascular complications & $88(8.2)$ & $187(7.2)$ & $149(5.3)$ & 0.001 \\
\hline Nonvascular in-lab complications & $43(4.0)$ & $87(3.3)$ & $87(3.1)$ & 0.386 \\
\hline Nonvascular postprocedural complications & $11(1.0)$ & $20(0.8)$ & $25(0.9)$ & 0.725 \\
\hline
\end{tabular}

Values are presented as $n(\%)$.

BMI: body mass index; NW: normal weight; OW: overweight; OB: obese; PCI: percutaneous coronary intervention.

${ }^{*} p$ values for chi-squared tests.

Vascular complications were defined as hematoma $(>5 \mathrm{~cm})$, pseudoaneurysm, arteriovenous fistula, vascular occlusion, access site bleeding, retroperitoneal bleed, loss of distal pulse, or occlusion.

Nonvascular complications occurring in-lab included abrupt coronary closure, emergency coronary artery bypass grafting (CABG), access site complications, death, ventricular tachycardia/ventricular fibrillation, pulmonary edema, shock, and dissection.

Nonvascular postprocedural complications included death, myocardial infarction, emergency CABG, abrupt coronary closure, hemorrhagic or ischemic CVA, and GI bleed.

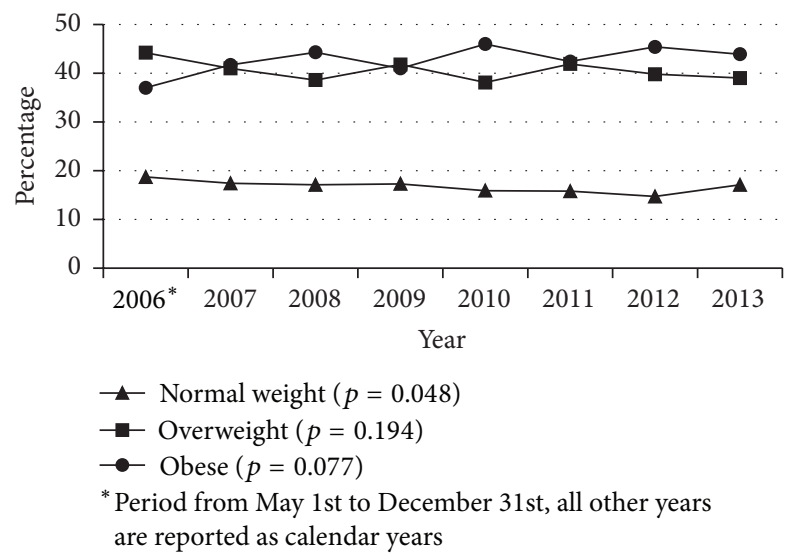

FIGURE 1: Body mass index trends for normal weight, overweight, and obese patients from 2006 to 2013.

angiotensin-converting enzyme inhibitor/angiotensin receptor blocker, calcium channel blockers, and long-acting nitrates.

3.2. Angiographic and Procedural Data. Admitting clinical, angiographic, and procedural data are shown in Table 3. Normal weight patients were significantly less likely to require a closure device $(p<0.001)$ compared to other BMI groups. However, there were no significant differences among the $\mathrm{BMI}$ categories in the prevalence of prior PCI, prior CABG, prior HF, prior MI, pulmonary embolism, thromboembolic history, deep vein thrombosis, same sitting angioplasty, IABP use at time of referral or during the procedure/cardiogenic shock at time of procedure, use of GP IIb/IIIa inhibitors, access site, and choice of sheath size. A greater proportion of normal weight patients presented as emergency/urgent cases, whereas more elective procedures were performed in overweight and obese patients. A greater proportion of obese patients presented with unstable angina, whereas a much lower proportion presented with a STEMI.

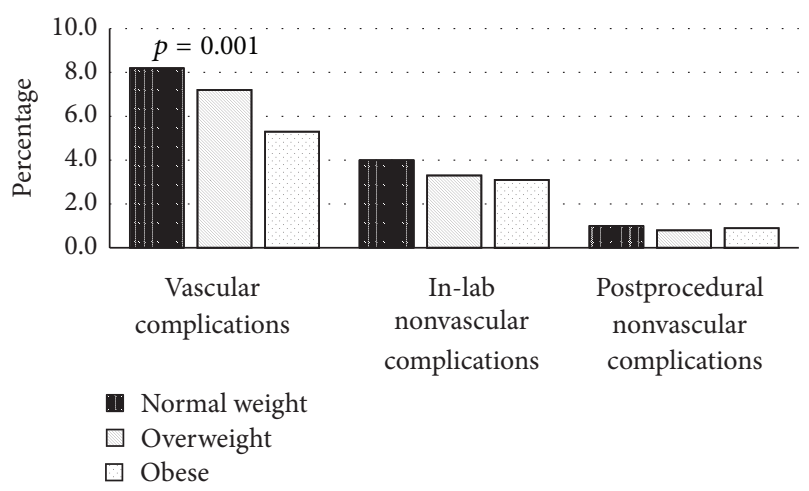

FIGURE 2: Prevalence of vascular and nonvascular complications (inlab and postprocedural) by body mass index category.

3.3. Complications Occurring within 24 to 48 Hours of PCI according to BMI. Complications occurring within 24 to 48 hours of PCI according to BMI category are presented in Table 4 and Figure 2. Obese subjects experienced a lower proportion of vascular complications (normal, overweight, and obese: $8.2 \%, 7.2 \%$, and $5.3 \%, p=0.001$ ). No significant differences were observed for nonvascular complications either in-lab $(4.0 \%, 3.3 \%$, and $3.1 \%, p=0.386)$ or postprocedural $(1.0 \%, 0.8 \%$, and $0.9 \%, p=0.725)$.

We performed multivariate analyses to adjust for clinical and procedural characteristics. Independent factors associated with the primary outcomes of vascular complications and nonvascular in-lab complications are shown in Tables 5 and 6. Increasing age, GP IIb/IIIa inhibitor, and LMWH use during the procedure and the utilization of a femoral access site approach were significant factors associated with the occurrence of vascular complications. Males, patients with diabetes and patients who had a closure device, and PCIs performed in 2010 were less likely to have vascular complications. GI/liver disease, coumadin use, utilization of GP IIb/IIIa inhibitors or IV heparin during PCI, and older age were significant factors associated with the occurrence of nonvascular in-lab complications. Male sex and the use of a closure device were protective factors associated with 
TABLE 5: Multivariate adjusted OR for vascular complications in patients undergoing PCI.

\begin{tabular}{lccc}
\hline & OR & $95 \%$ CI & $p$ value \\
\hline Age & 1.02 & $1.01-1.03$ & 0.001 \\
Male & 0.69 & $0.55-0.86$ & 0.001 \\
Diabetes & 0.65 & $0.51-0.84$ & 0.001 \\
Sheath size 5 Fr & 0.42 & $0.20-0.85$ & 0.016 \\
$\begin{array}{l}\text { Procedural GP IIb/IIIa } \\
\text { inhibitors }\end{array}$ & 1.95 & $1.50-2.54$ & 0.000 \\
$\begin{array}{l}\text { Preprocedural LMWH } \\
\text { Closure device }\end{array}$ & 1.29 & $1.03-1.61$ & 0.029 \\
Femoral access & 0.54 & $0.43-0.68$ & 0.000 \\
Year, 2010 & 2.98 & $2.00-4.45$ & 0.000 \\
BMI (referent category is & 0.49 & $0.30-0.80$ & 0.005 \\
normal weight) & & & \\
$\quad$ Overweight & & & 0.967 \\
$\quad$ Obese & 1.01 & $0.76-1.33$ & 0.219 \\
\hline Adusted for access & 0.83 & $0.62-1.11$ & \\
\hline
\end{tabular}

Adjusted for access site, age, aspirin, BMI, closure device, diabetes, gender, GI/liver disease, LMWH in-lab, preprocedural LMWH, GP IIb/IIIa inhibitors in-lab, preprocedural GP IIb/IIIa inhibitors, preprocedural IV heparin, prior CVD, prior HF, prior PCI, sheath size, smoking status, ticlopidine/clopidogrel, and year.

BMI: body mass index; CVD: cardiovascular disease; GP: glycoprotein; HF: heart failure; LMWH: low molecular weight heparin; PCI: percutaneous coronary intervention.

TABLE 6: Multivariate adjusted OR for nonvascular in-lab complications in patients undergoing PCI.

\begin{tabular}{lccc}
\hline & OR & $95 \%$ CI & $p$ value \\
\hline Age & 1.02 & $1.001-1.03$ & 0.039 \\
Male & 0.64 & $0.47-0.87$ & 0.005 \\
GI/liver disease & 1.53 & $1.02-2.27$ & 0.038 \\
GP IIb/IIIa inhibitors in-lab & 4.99 & $3.65-6.81$ & 0.000 \\
IV heparin in-lab & 1.70 & $1.14-2.52$ & 0.009 \\
Coumadin & 2.38 & $1.14-4.98$ & 0.021 \\
Closure device & 0.28 & $0.19-0.40$ & 0.000 \\
BMI (referent category is & & & \\
normal weight) & & & \\
$\quad \begin{array}{l}\text { Overweight } \\
\text { Obese }\end{array}$ & 0.93 & $0.62-1.38$ & 0.705 \\
& 0.54 & $0.58-1.32$ & 0.879
\end{tabular}

Adjusted for age, BMI, closure device, coumadin, diabetes, DVT, family history of premature CAD, GP IIb/IIIa inhibitors in-lab, gender, GI/liver disease, hypertension, IV heparin in-lab, LMWH in-lab, prior CABG, prior COPD, prior CVD, prior HF, prior PCI, prior PVD, pulmonary embolism, sheath size, and year.

BMI: body mass index; CAD: coronary artery disease; CABG: coronary artery bypass grafting; COPD: chronic obstructive pulmonary disease; CVD: cardiovascular disease; DVT: deep vein thrombosis; GP: glycoprotein; HF: heart failure; LMWH: low molecular weight heparin; PCI: percutaneous coronary intervention; PVD: peripheral vascular disease.

a less likelihood of nonvascular in-lab complications. BMI was not a significant factor associated with either vascular or nonvascular in-lab complications (Tables 5 and 6).

\section{Discussion}

The present study examined all adult patients who had a PCI procedure performed between 2006 and 2013 in one Canadian province to determine the prevalence of obesity in this patient population and trend in rates over time. A second objective was to examine the relationship between $\mathrm{BMI}$ and short-term vascular and nonvascular complications occurring within 48 hours and compare outcomes among three BMI categories (normal weight, overweight, and obese). The majority of patients $(84.3 \%)$ were either overweight or obese. Our study findings are comparable to other studies that have used PCI registries [10, 11,30]. In the current study, we found that over time there was a significant trend of decreasing prevalence for the normal weight category of patients undergoing PCI $(p=0.048)$. Similar to previous studies, the current study demonstrates that obese patients presented with more risk factors for CAD than overweight or normal weight patients. Obese patients were younger, diabetic, and hypertensive and had higher rates of hyperlipidemia and family history of CAD.

We hypothesized that BMI was an independent correlate of outcome in patients undergoing PCI; more specifically, obese patients would experience worse outcomes compared to normal and overweight patients. The obese patients in the present study were significantly younger and had higher incidence of coronary risk factors such as diabetes mellitus and hypertension and had a family history of coronary artery but based on the findings of the univariate analyses had a significantly lower rate of vascular complications (hematoma $(>5 \mathrm{~cm})$, pseudoaneurysm, arteriovenous fistula, vascular occlusion, access site bleeding, retroperitoneal bleed, loss of distal pulse, or occlusion) than their normal weight and overweight counterparts. There were no significant differences in the rates of nonvascular in-lab (acute coronary closure, emergency CABG, access site complications, death, ventricular tachycardia/ventricular fibrillation, pulmonary edema, shock, and dissection) and postprocedural complications (death, myocardial infarction, emergency CABG, abrupt coronary closure, hemorrhagic or ischemic CVA, and GI bleed) among the BMI categories.

After multiple logistic regression analysis, BMI was not a significant predictor of short-term outcomes (vascular complications or in-lab nonvascular complications). Our data regarding BMI in NL patients is consistent with one previous Canadian study but is contradictory to the findings of a 2009 study conducted by Byrne et al. [28]. Similar to our findings, Shubair et al. [10] evaluated the effect of BMI on in-hospital outcomes in a consecutive series of coronary artery disease patients undergoing PCI enrolled in a clinical database at the Hamilton Health Sciences in Ontario, Canada. The authors found that obesity was not associated with inhospital postprocedural death, myocardial infarction, repeat PCI, CABG, or major adverse cardiac event defined as a composite of death, myocardial infarction, repeat PCI, and CABG. Using a large Canadian provincial registry, Byrne et al. [28] investigated the relationship between BMI, bleeding, and outcome (i.e., 1-year mortality) after PCI. The authors reported that lower BMI $\left(\leq 18.5 \mathrm{~kg} / \mathrm{m}^{2}\right)$ and higher BMI 
$\left(\geq 40 \mathrm{~kg} / \mathrm{m}^{2}\right)$ patients were at greater risk of bleeding and death after PCI. Other studies conducted in the western society have reported that underweight $[9,25,28]$, normal weight $[9,25]$, and extremely obese [29-31] patients are at greater risk of adverse outcomes after PCI. Cox et al. [9] reported that the rate of vascular complications was the highest in extremely thin and morbidly obese patients and the lowest in moderately obese patients. In a study by Gruberg et al. [25], the authors reported that normal weight patients were at the highest risk of in-hospital complications (i.e., major bleeding, vascular complications, emergency CABG, and myocardial infarction) and cardiac death compared to overweight and obese patients. Two studies by Gurm et al. $[26,27]$ suggested that being moderately obese conferred a protective effect, referred to as an "obesity paradox," in relation to vascular complications and major adverse outcomes after PCI, a finding consistent with that reported by Cox et al. [9].

In other studies that have focused primarily on the comparison of normal weight and extremely obese $\left(\geq 40 \mathrm{~kg} / \mathrm{m}^{2}\right)$ patients undergoing PCI, researchers have reported that extremely obese patients have increased vascular complications [30] compared to normal weight individuals and higher rates of in-hospital mortality [29-31] compared to overweight individuals. In the current study, we were unable to examine the various classes of obesity due to the small numbers in each category.

Study Strengths and Limitations. Our study has a number of strengths. We report on a large population-based cohort of patients undergoing PCI at a single tertiary cardiac centre using APPROACH-NL prospectively collected data. Data quality assurance indicated that the amount of missing data was minimal (1.7\%). Actual measures of height and weight were taken at the time of the procedure unless the patients were unstable.

This study also has a number of limitations. Our study is an observational nonrandomized cohort study with retrospective analysis. The current study design can only establish association and not causation. We used data from a clinical database and as such cannot account for confounders not captured in the database. The study population was heterogeneous (i.e., included patients with variable levels of coronary artery disease severity ranging from acute coronary syndrome with cardiogenic shock to stable angina). Patients with missing BMI data were excluded $(n=113)$ which may contribute to selection bias, but as missing data only accounted for $1.7 \%$ this is unlikely. Despite its widespread use, the use of BMI in terms of its accuracy to define obesity is controversial [37-39]. BMI is not as well correlated to cardiovascular disease and death as other measures including waist circumference and waist-to-hip ratio [40], data that were unavailable in the clinical database. A lack of underweight and severely obese patients meant that comparisons in our study were made between only three BMI groups: normal weight, overweight, and obese.

\section{Conclusion}

Overweight and obesity were not independent predictors of short-term outcomes (vascular or nonvascular complications occurring within 24 to 48 hours) in patients undergoing PCI at our institution.

\section{Competing Interests}

Dr. Anne B. Gregory, Kendra K. Lester, Dr. Deborah M. Gregory, Dr. Laurie K. Twells, and Dr. William K. Midodzi have no competing interests to declare. Dr. Neil J. Pearce is the director of the APPROACH-NL cardiac care database, Division Head Cardiology, Eastern Health, and the former director of the Cardiac Catheterization Lab.

\section{Authors' Contributions}

Study concept and design were handled by Anne B. Gregory, Deborah M. Gregory, Laurie K. Twells, and Neil J. Pearce. Analysis and interpretation of the data were handled by Anne B. Gregory, Kendra K. Lester, Deborah M. Gregory, Neil J. Pearce, Laurie K. Twells, and William K. Midodzi. Drafting of the paper was handled by Anne B. Gregory and Deborah M. Gregory. Critical revision of the paper for important intellectual content was handled by Anne B. Gregory, Deborah M. Gregory, Kendra K. Lester, Neil J. Pearce, Laurie K. Twells, and William K. Midodzi. Statistical analysis was carried out by Anne B. Gregory, Deborah M. Gregory, Kendra K. Lester, and William K. Midodzi. Anne B. Gregory, Deborah M. Gregory, Kendra K. Lester, and William K. Midodzi had full access to all data in the study and take responsibility for the integrity of the data and the accuracy of the data analysis.

\section{Acknowledgments}

The authors gratefully acknowledge the cardiac care program staff for data collection and entry, especially Jennifer Matthews, Program Coordinator of APPROACH-NL, and staff from the Translational Personalized Medicine Initiative of Memorial University for their statistical consultation.

\section{References}

[1] S. W. Rabkin, F. A. L. Mathewson, and P.-H. Hsu, "Relation of body weight to development of ischemic heart disease in a cohort of young North American men after a 26 year observation period: the Manitoba study," The American Journal of Cardiology, vol. 39, no. 3, pp. 452-458, 1977.

[2] J. E. Manson, G. A. Colditz, M. J. Stampfer et al., "A prospective study of obesity and risk of coronary heart disease in women," The New England Journal of Medicine, vol. 322, no. 13, pp. 882889, 1990.

[3] H. B. Hubert, M. Feinleib, P. M. McNamara, and W. P. Castelli, "Obesity as an independent risk factor for cardiovascular disease: a 26-year follow-up of participants in the Framingham Heart Study," Circulation, vol. 67, no. 5, pp. 968-977, 1983. 
[4] Y. Chen, W. K. Copeland, R. Vedanthan et al., "Association between body mass index and cardiovascular disease mortality in east Asians and south Asians: pooled analysis of prospective data from the Asia Cohort Consortium," British Medical Journal, vol. 347, no. 7927, Article ID f5446, 2013.

[5] Y. Lu, K. Hajifathalian, M. Ezzati, M. Woodward, E. B. Rimm, and G. Danaei, "Metabolic mediators of the effects of bodymass index, overweight, and obesity on coronary heart disease and stroke: a pooled analysis of 97 prospective cohorts with 1.8 million participants," The Lancet, vol. 383, no. 9921, pp. 970-983, 2014.

[6] K. R. Fontaine, D. T. Redden, C. Wang, A. O. Westfall, and D. B. Allison, "Years of life lost due to obesity," The Journal of the American Medical Association, vol. 289, no. 2, pp. 187-193, 2003.

[7] E. E. Calle, M. J. Thun, J. M. Petrelli, C. Rodriguez, and C. W. Heath Jr., "Body-mass index and mortality in a prospective cohort of U.S. adults," The New England Journal of Medicine, vol. 341, no. 15, pp. 1097-1105, 1999.

[8] K. M. Flegal, B. K. Kit, H. Orpana, and B. I. Graubard, "Association of all-cause mortality with overweight and obesity using standard body mass index categories: a systematic review and meta-analysis," The Journal of the American Medical Association, vol. 309, no. 1, pp. 71-82, 2013.

[9] N. Cox, F. S. Resnic, J. J. Popma, D. I. Simon, A. C. Eisenhauer, and C. Rogers, "Comparison of the risk of vascular complications associated with femoral and radial access coronary catheterization procedures in obese versus nonobese patients," The American Journal of Cardiology, vol. 94, no. 9, pp. 1174-1177, 2004.

[10] M. M. Shubair, P. Prabhakaran, V. Pavlova, J. L. Velianou, A. M. Sharma, and M. K. Natarajan, "The relationship of body mass index to outcomes after percutaneous coronary intervention," Journal of Interventional Cardiology, vol. 19, no. 5, pp. 388-395, 2006.

[11] L. Mehta, W. Devlin, P. A. McCullough et al., "Impact of body mass index on outcomes after percutaneous coronary intervention in patients with acute myocardial infarction," American Journal of Cardiology, vol. 99, no. 7, pp. 906-910, 2007.

[12] C. E. Hastie, S. Padmanabhan, R. Slack et al., "Obesity paradox in a cohort of 4880 consecutive patients undergoing percutaneous coronary intervention," European Heart Journal, vol. 31, no. 2, pp. 222-226, 2010.

[13] T. Lancefield, D. J. Clark, N. Andrianopoulos et al., "Is there an obesity paradox after percutaneous coronary intervention in the contemporary era? An analysis from a multicenter australian registry," Journal of the American College of Cardiovascular Interventions, vol. 3, no. 6, pp. 660-668, 2010.

[14] M. Schmiegelow, C. Torp-Pedersen, G. H. Gislason et al., "Relation of body mass index to risk of stent thrombosis after percutaneous coronary intervention," The American Journal of Cardiology, vol. 110, no. 11, pp. 1592-1597, 2012.

[15] S. Uretsky, F. H. Messerli, S. Bangalore et al., "Obesity paradox in patients with hypertension and coronary artery disease," American Journal of Medicine, vol. 120, no. 10, pp. 863-870, 2007.

[16] G. C. Fonarow, P. Srikanthan, M. R. Costanzo, G. B. Cintron, and M. Lopatin, "An obesity paradox in acute heart failure: analysis of body mass index and inhospital mortality for 108,927 patients in the Acute Decompensated Heart Failure National Registry," American Heart Journal, vol. 153, no. 1, pp. 74-81, 2007.

[17] A. Oreopoulos, R. Padwal, C. M. Norris, J. C. Mullen, V. Pretorius, and K. Kalantar-Zadeh, "Effect of obesity on short- and long-term mortality postcoronary revascularization: a metaanalysis," Obesity, vol. 16, no. 2, pp. 442-450, 2008.

[18] A. Romero-Corral, V. M. Montori, V. K. Somers et al., "Association of bodyweight with total mortality and with cardiovascular events in coronary artery disease: a systematic review of cohort studies," The Lancet, vol. 368, no. 9536, pp. 666-678, 2006.

[19] A. Oreopoulos, F. A. McAlister, K. Kalantar-Zadeh et al., "The relationship between body mass index, treatment, and mortality in patients with established coronary artery disease: a report from APPROACH," European Heart Journal, vol. 30, no. 21, pp. 2584-2592, 2009.

[20] A. P. Johnson, J. L. Parlow, M. Whitehead, J. Xu, S. Rohland, and B. Milne, "Body mass index, outcomes, and mortality following cardiac surgery in Ontario, Canada," Journal of the American Heart Association, vol. 4, no. 7, Article ID e002140, 2015.

[21] K. Kalantar-Zadeh, G. Block, M. H. Humphreys, and J. D. Kopple, "Reverse epidemiology of cardiovascular risk factors in maintenance dialysis patients," Kidney International, vol. 63, no. 3, pp. 793-808, 2003.

[22] K. Kalantar-Zadeh, G. Block, T. Horwich, and G. C. Fonarow, "Reverse epidemiology of conventional cardiovascular risk factors in patients with chronic heart failure," Journal of the American College of Cardiology, vol. 43, no. 8, pp. 1439-1444, 2004.

[23] J. D. Kopple, “The phenomenon of altered risk factor patterns or reverse epidemiology in persons with advanced chronic kidney failure," The American Journal of Clinical Nutrition, vol. 81, no. 6, pp. 1257-1266, 2005.

[24] N. W. Levin, G. J. Handelman, J. Coresh, F. K. Port, and G. A. Kaysen, "Reverse epidemiology: a confusing, confounding, and inaccurate term," Seminars in Dialysis, vol. 20, no. 6, pp. 586592, 2007.

[25] L. Gruberg, N. J. Weissman, R. Waksman et al., "The impact of obesity on the short-term and long-term outcomes after percutaneous coronary intervention: the obesity paradox?" Journal of the American College of Cardiology, vol. 39, no. 4, pp. 578-584, 2002.

[26] H. S. Gurm, D. M. Brennan, J. Booth, J. E. Tcheng, A. M. Lincoff, and E. J. Topol, "Impact of body mass index on outcome after percutaneous coronary intervention (The Obesity Paradox)," The American Journal of Cardiology, vol. 90, no. 1, pp. 42-45, 2002.

[27] H. S. Gurm, P. L. Whitlow, and K. E. Kip, "The impact of body mass index on short- and long-term outcomes in patients undergoing coronary revascularization. Insights from the Bypass Angioplasty Revascularization Investigation (BARI)," Journal of the American College of Cardiology, vol. 39, no. 5, pp. 834-840, 2002.

[28] J. Byrne, M. S. Spence, E. Fretz et al., "Body mass index, periprocedural bleeding, and outcome following percutaneous coronary intervention (from the British Columbia Cardiac Registry)," The American Journal of Cardiology, vol. 103, no. 4, pp. 507-511, 2009.

[29] S. R. Das, K. P. Alexander, A. Y. Chen et al., "Impact of body weight and extreme obesity on the presentation, treatment, and in-hospital outcomes of 50,149 patients with ST-segment elevation myocardial infarction: results from the NCDR (National Cardiovascular Data Registry)," Journal of the American College of Cardiology, vol. 58, no. 25, pp. 2642-2650, 2011.

[30] M. E. Buschur, D. Smith, D. Share et al., "The burgeoning epidemic of morbid obesity in patients undergoing percutaneous coronary intervention: insight from the blue cross blue shield of 
Michigan cardiovascular consortium," Journal of the American College of Cardiology, vol. 62, no. 8, pp. 685-691, 2013.

[31] S. Payvar, S. Kim, S. V. Rao et al., "In-hospital outcomes of percutaneous coronary interventions in extremely obese and normal-weight patients. Findings from the NCDR (National Cardiovascular Data Registry)," Journal of the American College of Cardiology, vol. 62, no. 8, pp. 692-696, 2013.

[32] L. K. Twells, D. M. Gregory, J. Reddigan, and W. K. Midodzi, "Current and predicted prevalence of obesity in Canada: a trend analysis," CMAJ Open, vol. 2, no. 1, pp. E18-E26, 2014.

[33] D. Gregory, W. K. Midodzi, and N. J. Pearce, "Complications with Angio-Seal ${ }^{\mathrm{TM}}$ vascular closure devices compared with manual compression after diagnostic cardiac catheterization and percutaneous coronary intervention," Journal of Interventional Cardiology, vol. 26, no. 6, pp. 630-638, 2013.

[34] WHO Consultation on Obesity, "Obesity: preventing and managing the global epidemic. Report of a WHO consultation," World Health Organization Technical Report Series 894, WHO, 2000.

[35] Health Canada, "Canadian guidelines for body weight classification in adults. Quick reference tool for professionals," 2003, http://www.hc-sc.gc.ca/fn-an/nutrition/weights-poids/guide-ldadult/cg_quick_ref-ldc_rapide_ref-table1-eng.php.

[36] IBM SPSS Statistics for Windows, Version 22.0, IBM Corp, Armonk, NY, USA, 2013.

[37] A. Romero-Corral, V. K. Somers, J. Sierra-Johnson et al., "Diagnostic performance of body mass index to detect obesity in patients with coronary artery disease," European Heart Journal, vol. 28, no. 17, pp. 2087-2093, 2007.

[38] A. Romero-Corral, V. K. Somers, J. Sierra-Johnson et al., "Accuracy of body mass index in diagnosing obesity in the adult general population," International Journal of Obesity, vol. 32, no. 6, pp. 959-966, 2008.

[39] A. De Schutter, C. J. Lavie, K. Arce, S. G. Menendez, and R. V. Milani, "Correlation and discrepancies between obesity by body mass index and body fat in patients with coronary heart disease," Journal of Cardiopulmonary Rehabilitation and Prevention, vol. 33, no. 2, pp. 77-83, 2013.

[40] M. Chrostowska, A. Szyndler, M. Hoffmann, and K. Narkiewicz, "Impact of obesity on cardiovascular health," Best Practice and Research: Clinical Endocrinology and Metabolism, vol. 27, no. 2, pp. 147-156, 2013. 


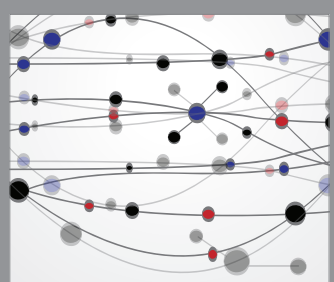

The Scientific World Journal
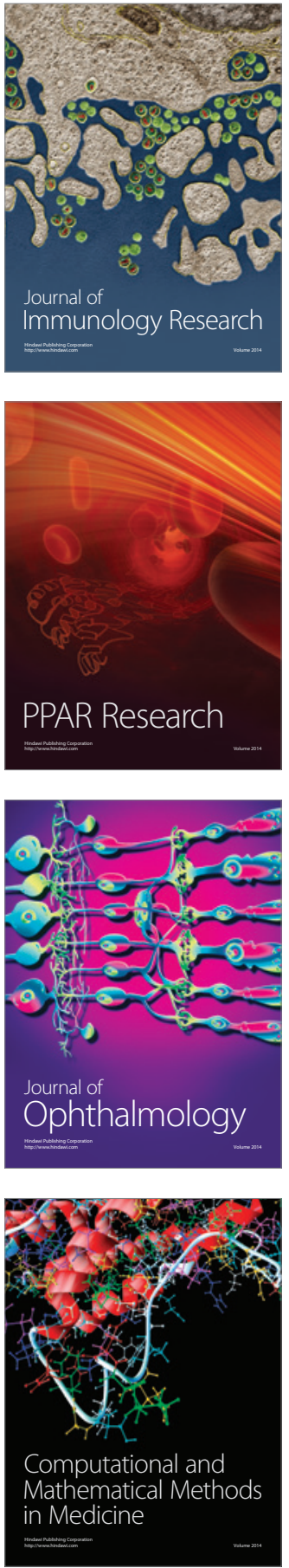

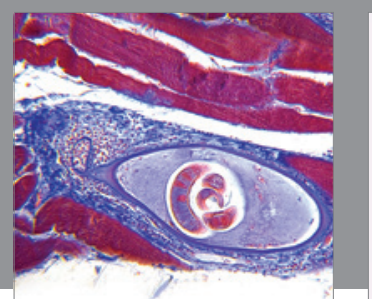

Gastroenterology Research and Practice

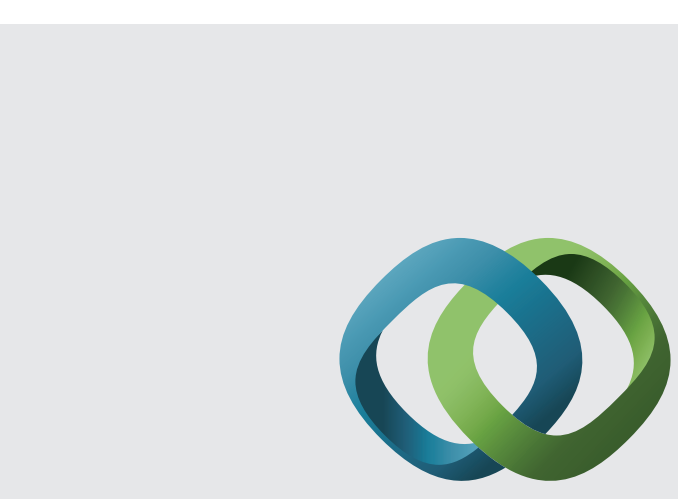

\section{Hindawi}

Submit your manuscripts at

http://www.hindawi.com
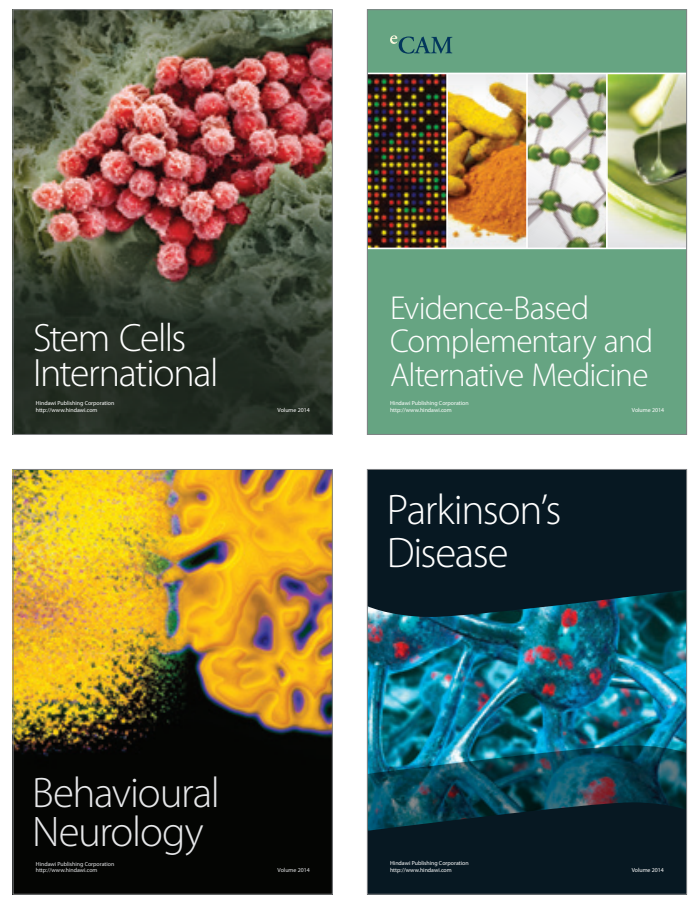
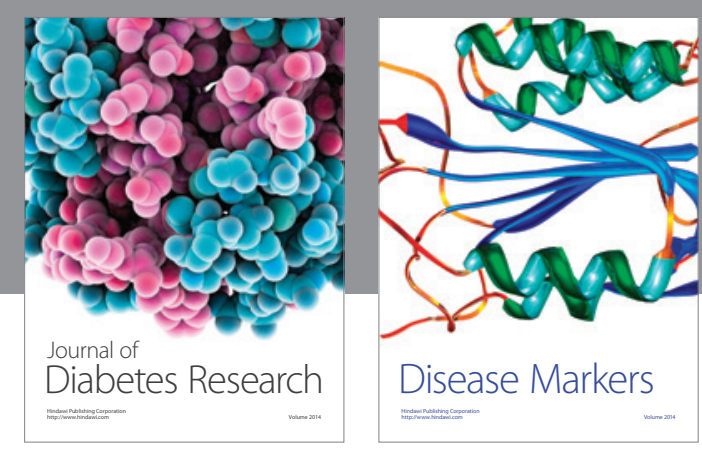

Disease Markers
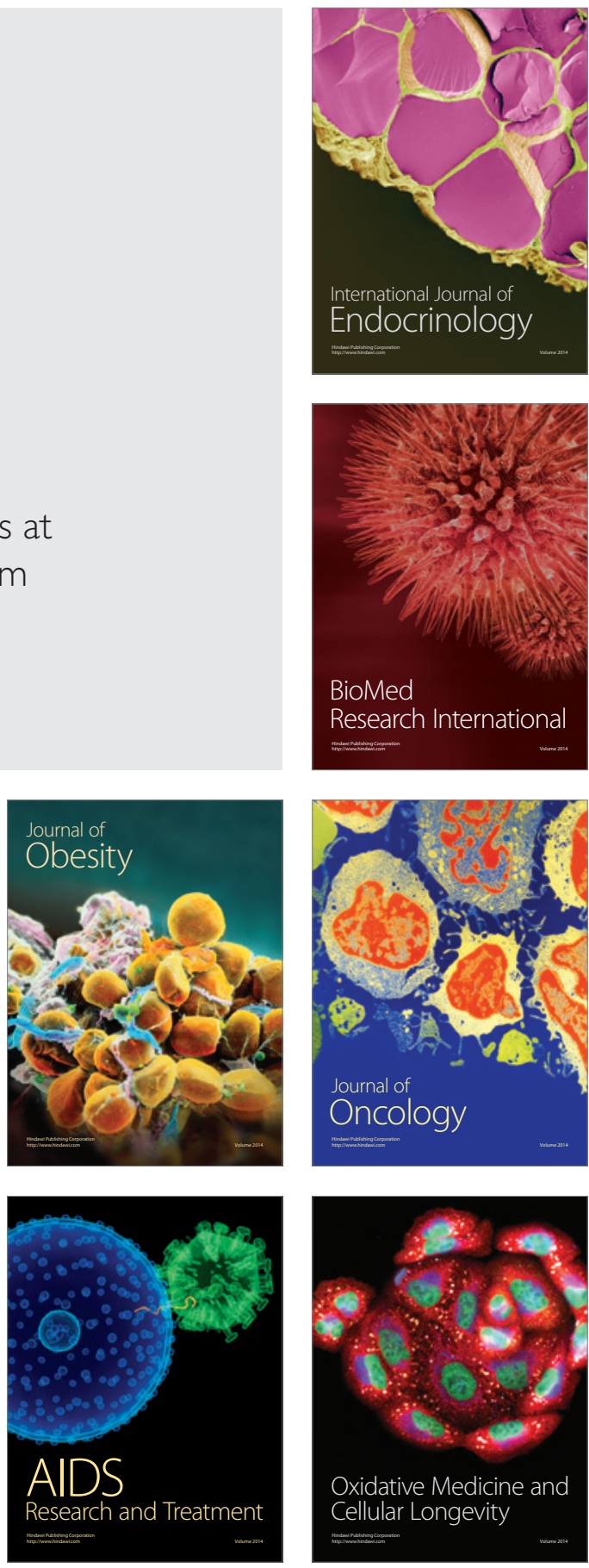